\title{
Improved Small-Angle Sensor Based on Total-Internal Reflection and Surface Plasmon Resonance in Heterodyne Interferometry
}

\author{
Shinn-Fwu Wang*, Hung-Shing Tsai, Yi Chu, Jeng-Hua Wei, \\ Yuan-Fong Chau, An-Li Liu and Fu-Hsi Kao \\ Department of Electronic Engineering, Chien Hsin University of Science and Technology, \\ No. 229, Jianxing Rd., Zhongli City, Taoyuan County 32097, Taiwan
}

(Received November 5, 2012; accepted February 4, 2013)

Key words: small-angle measurement, total-internal reflection (TIR), heterodyne interferometry (HI), surface plasmon resonance (SPR), Kretchmann's configuration

In this paper, an improved small-angle sensor based on total-internal reflection (TIR) and surface plasmon resonance (SPR) in heterodyne interferometry (HI) is proposed. The improved small-angle sensor is designed as a reflective elongated prism made of BK7 glass. The shorter-side surface of the reflective elongated prism was coated with a 2 $\mathrm{nm}$ Ti film and a $45.5 \mathrm{~nm}$ Au film, but the longer-side surface was not coated with metal films. With the new small-angle sensor, a small rotation angle can be obtained simply by measuring the phase difference between the p-and s-polarization lights owing to the effects of multiple attenuated total reflections (ATRs) and TIRs. Its angular resolution can reach $2.95 \times 10^{-7} \mathrm{rad}$ at least. Moreover, the improved small-angle sensor is very stable because its common optical path is insensitive to environmental disturbances. The improved small-angle sensor has some advantages, such as high resolution, high sensitivity, stability, and real-time testing.

\section{Introduction}

Owing to the importance of the small-angle measurement and the application of quaternion in rotation, there have been many $\operatorname{articles}^{(1-7)}$ about them over the past few decades. Chiu and coworkers ${ }^{(1,2)}$ have presented some improved techniques of small-angle measurement by modifying the optical systems proposed by Huang and coworkers. ${ }^{(3,4)}$ They tried to improve the resolution of such systems using two parallelogram prisms as the small-angle-sensing unit instead of two right-angle prisms. The angular resolution of the system proposed by Chiu and coworkers was better than $2.2 \times 10^{-6} \mathrm{rad}$. Previously, Wang and coworkers proposed some methods of small-

${ }^{*}$ Corresponding author: e-mail: sfwang@uch.edu.tw 
angle measurement based on the surface plasmon resonance (SPR) technology and heterodyne interferometry $(\mathrm{HI}){ }^{(5,6)}$ Although the resolutions of some of these reported systems are high, the signals measured by these systems are very weak around the resonant angle owing to the attenuated total reflection (ATR) effect. Moreover, the plots of resolution versus the rotation angle obtained by the above-mentioned methods are obviously nonlinear, and the methods could not reach a wide measurement range and high resolution simultaneously. In this paper, the initial incident angle of the smallangle-sensing unit is not equal to the resonant angle. Thus, the measured signal will not be weakened by the ATR effect. Note that the sensor can reach a high resolution owing to multiple ATR and total-internal reflection (TIR) effects. It is for this reason that we propose the improved small-angle sensor in this paper to improve the linearity of the phase variation versus the small rotation angle.

The improved small-angle sensor is designed as a reflective elongated prism $^{(7)}$ that is based on the method previously reported by Wang and coworkers. With the improved small-angle sensor, the small rotation angle can be obtained only by measuring the phase difference between the p-and s-polarization lights due to the ATR and TIR effects. The angular resolution of the improved sensor is better than $2.95 \times 10^{-7}$ rad over the measurement range of $-0.05^{\circ} \leq \Delta \theta \leq 0.05^{\circ}$, where $\Delta \theta$ is the rotation angle. Moreover, its feasibility has been demonstrated.

\section{Principles}

As heterodyne light ${ }^{(2)}$ is transmitted from a dense medium $\left(n_{1}\right)$ through a sparse medium $\left(n_{2}\right)$, the refractive light will deviate from the normal line when the incident angle increases gradually. When the incident angle reaches the critical angle $\theta_{c}$, the refractive light is transmitted along the boundary of the medium. As shown in Fig. 1, the TIR effect will occur if a heterodyne light is incident on the interface between medium 1 and medium 2 at the incident angle $\theta_{\mathrm{i}}, \theta_{\mathrm{i}}>\theta_{\mathrm{c}}$, where $\theta_{\mathrm{c}}=\tan ^{-1}\left(n_{1} / n_{2}\right)$ and $n_{1}>n_{2}$. According to Fresnel's equations, ${ }^{(2)}$ the phase difference $\varphi_{1}$ between s- and p-polarizations is given as ${ }^{(8,9)}$

$$
\varphi_{1}=\varphi_{\mathrm{s}}-\varphi_{\mathrm{p}}=2 \tan ^{-1}\left\{\frac{\sqrt{\sin ^{2}\left[45^{\circ}+\sin ^{-1}\left(\frac{\sin \theta}{n}\right)-\frac{1}{n^{2}}\right]}}{\tan \left[45^{\circ}+\sin ^{-1}\left(\frac{\sin \theta}{n}\right)\right] \sin \left[45^{\circ}+\sin ^{-1}\left(\frac{\sin \theta}{n}\right)\right]}\right\},
$$

where $\varphi_{\mathrm{s}}$ and $\varphi_{\mathrm{p}}$ are the phases of s- and p-polarization components, respectively.

For the four-layer Kretchmann's configuration ${ }^{(10)}$ (BK7 glass prism-Ti-Au-air) shown in Fig. 2, surface plasmons are excited when $\alpha$ equals the resonant angle $\alpha_{\mathrm{sp}}$. From Maxwell's equations, the reflectivities of p- and s-polarizations can be expressed as ${ }^{(8)}$ $r_{1234}^{\mathrm{p}}=\left|r_{1234}^{\mathrm{p}}\right| e^{i \varphi_{\mathrm{p}}}$ and $r_{1234}^{\mathrm{s}}=\left|r_{1234}^{\mathrm{s}}\right| e^{i \varphi_{\mathrm{s}}}$, respectively. We can obtain the phase difference variation $\varphi_{2}$ between s- and p-polarization components as $\varphi_{2}=\varphi_{\mathrm{s}}-\varphi_{\mathrm{p}}$. Moreover, the reflectivities of $\mathrm{p}$ - and s-polarization components are $R_{\mathrm{p}}=\left|r_{1234}^{\mathrm{p}}\right|^{2}$ and $R_{\mathrm{s}}=\left|r_{1234}^{\mathrm{s}}\right|^{2}$, respectively.

In this paper, the parameters of the four-layer device (BK7 glass prism-Ti-Au-air) are a Ti film thickness $d_{2}$ of $2 \mathrm{~nm}$, a Au film thickness $d_{3}$ of $45.5 \mathrm{~nm}$, and a wavelength $\lambda$ of 

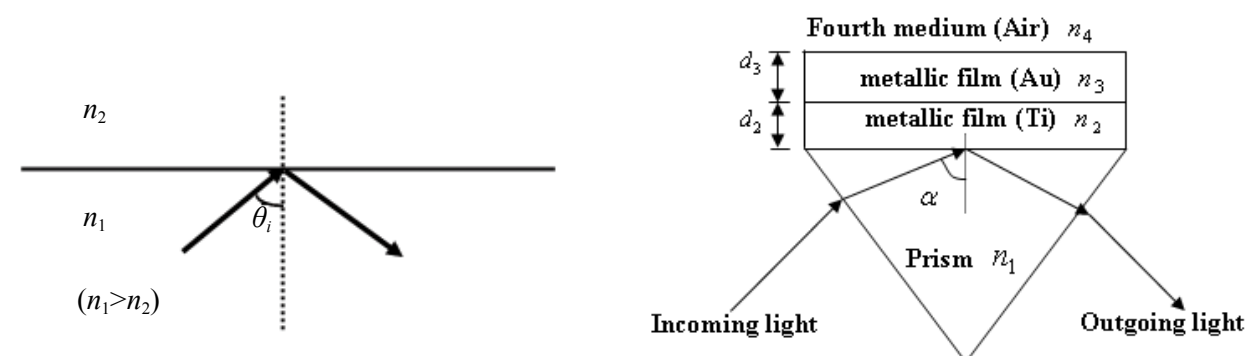

Fig. 1 (left). TIR effect.

Fig. 2 (right). Four-layer Kretchmann's configuration.

$632.8 \mathrm{~nm}$. Figure 3 shows the plot of the reflectivity of p-polarization light versus the incident angle $\alpha$ in the range of $40^{\circ} \leq \alpha \leq 45^{\circ}$. It is evident from Fig. 3 that reflectivity varies evidently around the resonant angle $\alpha_{\mathrm{sp}}\left(\alpha_{\mathrm{sp}} \approx 43.85^{\circ}\right)$.

In this article, a refractive elongated prism is proposed as the new small-angle sensing unit (Fig. 4). First, the refractive elongated prism is mounted on a rotation stage. When a laser light enters into the elongated prism, it will undergo multiple total-internal reflections and attenuated total reflections.

In order to obtain the output signal of the sensor, we choose $\theta=\theta_{0}=-2.2723^{\circ}$ as the initial angle at the hypotenuse of the elongated prism. At this moment, the light is incident on the two side surfaces of the reflective elongated prism at $\theta_{1}=\theta_{10}=43.5^{\circ}$. As we rotate the rotation stage at an angle, the incident angle $\theta_{1}$ is equal to $\theta_{10}+\Delta \theta_{1}$; we can obtain

$$
\begin{gathered}
\theta=\theta_{10}+\Delta \theta_{1}=45^{\circ}+\theta^{\prime}, \\
1 \times \sin \left(\theta_{i}\right)=n \times \sin \left(\theta^{\prime}\right), \\
l=h \times \tan \left(45^{\circ}+\theta^{\prime}\right), \\
m_{1} \leq(L / l)<\left(m_{1}+1\right), \\
m_{2} \leq(D / l)<\left(m_{2}+1\right),
\end{gathered}
$$

where $l$ is the reflection distances between two adjacent points along the $z$-axis, $n$ is the refractive index of the elongated prism, $L$ is the length of the longer side of the elongated prism, $m_{1}$ is the TIR times, and $m_{2}$ is the ATR times. The parameters of the sensor are $D=100 \mathrm{~mm}, L=110 \mathrm{~mm}$, and $h=10 \mathrm{~mm}$. From eqs. (2)-(6), the heterodyne light will undergo 11 ATR times on the shorter-side surface of the elongated prism and 12 TIR times on the longer-side of that. In addition, there is one TIR time on the end-side surface of the elongated prism. 

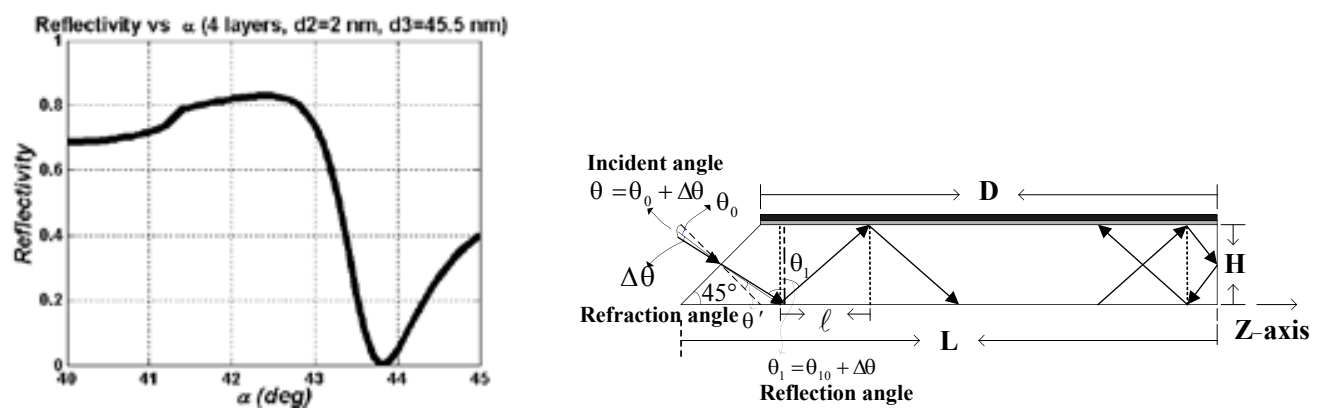

Fig. 3 (left). Reflectivity of p-polarization light vs $\alpha$.

Fig. 4 (right). Small-angle sensing unit.

As the rotation stage rotates at a small angle $\Delta \theta$, we can obtain the phase difference $\varphi$ between $\mathrm{s}-$ and $\mathrm{p}$-polarization components, and $\varphi$ is given by

$$
\varphi_{1}=m_{1} \varphi_{1}+m_{2} \varphi_{2}+\varphi_{3}
$$

where $m_{1}=12, m_{2}=11$, and $\varphi_{3}$ is the phase difference due to the TIR effect on the endside surfaces of the elongated prism.

\section{Experiment and Results}

The experimental setup of the system is shown in Fig. 5. In the experimental configuration, the new small-angle sensor is mounted on a precision rotary stage with a resolution of $0.0002^{\circ}$, and a heterodyne optical source with $2 \mathrm{kHz}$ beat frequency is used. The heterodyne optical source ${ }^{(2)}$ is composed of a He-Ne laser, a polarizer, and an electro-optical modulator (E-O modulator). As the heterodyne light passes through a half-wave plate and is incident on a beamsplitter (BS), the reflected light passes through the analyzer $\mathrm{AN}_{\mathrm{r}}$ and enters the photodetector $\mathrm{PD}_{\mathrm{r}}$. The signal measured by $\mathrm{PD}_{\mathrm{r}}$ is the reference signal. The transmitted light from the BS enters the improved small-angle sensor and undergoes ATRs and TIRs. Then the light reflects back from the end surface of the elongated prism. Finally, the light propagates out of the sensor and passes through the analyzer $\mathrm{AN}_{\mathrm{t}}$. We can obtain the test signal using the photodetector $\mathrm{PD}_{\mathrm{t}}$. These two signals are sent to a lock-in amplifier (LIA; SR830, manufactured by Stanford Research Systems, Sunnyvale, California) with the resolution $\Delta \Phi$ of $0.01^{\circ}$. Thus, we can obtain the phase difference due to the ATR and TIR effects. After some numerical computations using a computer, a small rotation angle can be measured.

Figure 6 shows the experimental curve of the phase difference versus the rotating angle $\Delta \theta$ of the rotation stage. It is evident that only evaluating the phase difference variation is necessary for small-angle measurement. Moreover, the experimental results and theoretical curve show good agreement. 

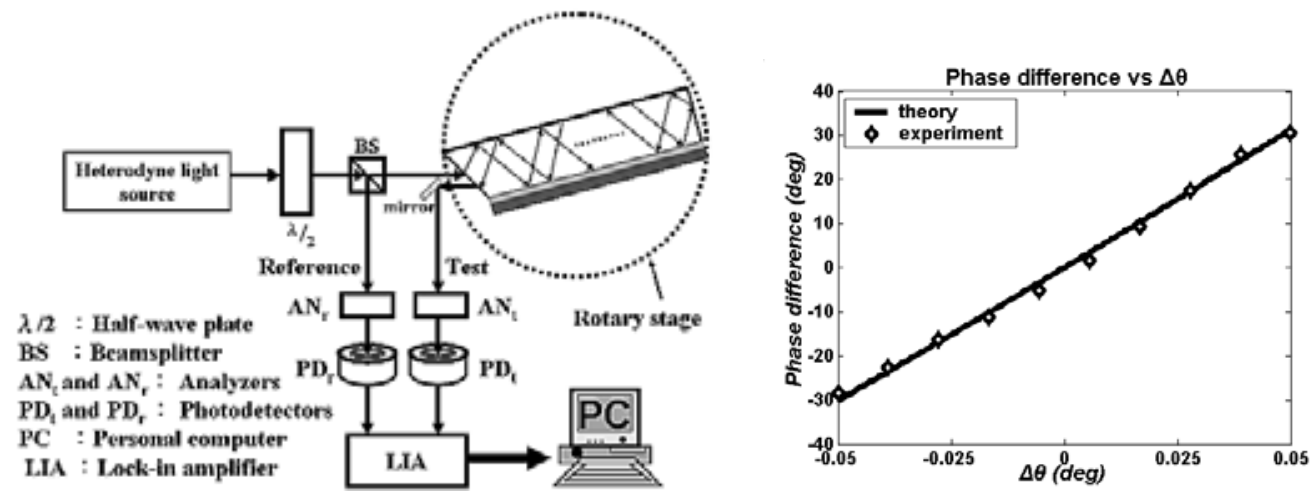

Fig. 5 (left). Experimental setup.

Fig. 6 (right). Experimental and simulation results.

\section{Discussions}

In this study, a small rotation angle $(\Delta \theta)$ can be obtained simply by measuring the phase difference variation using a heterodyne interferometer. First, let us discuss the resolution of the new small-angle sensor. The resolution $R$ of the system is defined as

$$
R=\frac{\mathrm{d} \theta}{\mathrm{d} \varphi} \Delta \Phi
$$

where $\mathrm{d} \varphi$ is the variation of the phase difference, $\Delta \Phi$ is the resolution of the lockin amplifier, and $\mathrm{d} \theta$ is the small-angle change made by the rotation of the rotary stage. As shown in Fig. 7, the resolution of the sensor will reach $2.95 \times 10^{-7}$ rad over the measurement range of $-0.05^{\circ} \leq \Delta \theta \leq 0.05^{\circ}$. It is evident that the phase difference variation is almost proportional to the small rotation angle. Moreover, the experimental results and theoretical curve show good agreement. In addition, the experimental phase error (\%) versus the displacement $\Delta z$ in Fig. 8 shows that the phase error is less than $2 \%$ in the measurement range of $-0.05^{\circ} \leq \Delta \theta \leq 0.05^{\circ}$.

\section{Conclusions}

In the paper, an improved small-angle sensor based on TIR and SPR in HI is presented. With the small-angle sensor, a small rotation angle $(\Delta \theta)$ can be detected simply by evaluating the phase difference variation $(\Delta \theta)$ between s- and p-polarizations due to the multiple TIRs and ATRs. From the experimental results, we observe that the phase difference variations are almost proportional to $\Delta \theta$. In addition, the improved small-angle sensor is very stable because of its common optical path insensitive to environmental disturbances. Moreover, the improved small-angle sensor has some merits, e.g., a simple optical setup, easy operation, high measurement accuracy, high resolution, rapid measurement, and high stability. We can design a nanometer-scale small-displacement sensor ${ }^{(8)}$ using the improved small-angle sensor in the future. 

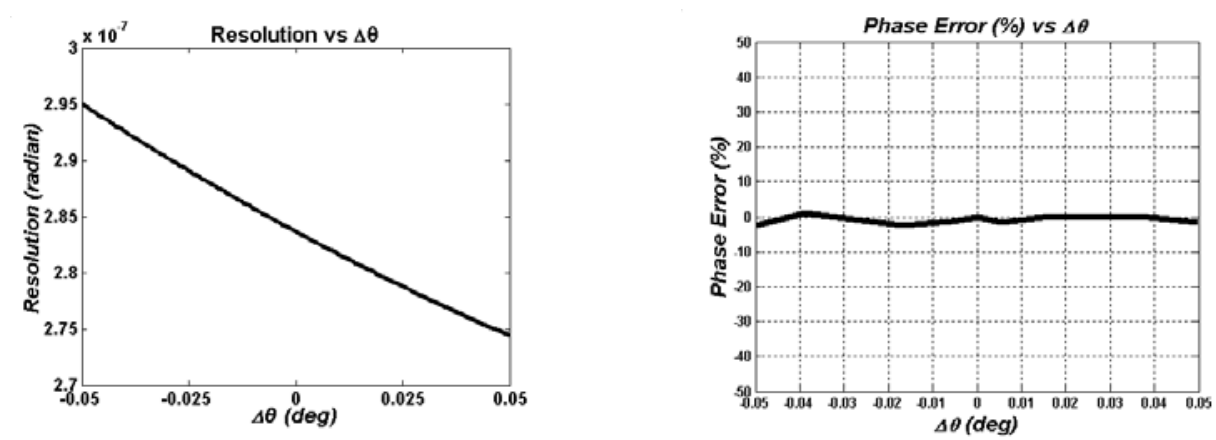

Fig. 7 (left). Resolution vs $\Delta \theta$.

Fig. 8 (right). Relative phase error (\%) vs $\Delta \theta$.

\section{Acknowledgements}

This research was partially supported by the National Science Council in Taiwan through grant Nos. NSC 100-2632-E-231-001-MY3 and NSC 97-2221-E-231-001-MY2.

\section{References}

1 M. H. Chiu and D. C. Su: Appl. Opt. 36 (1997) 7104.

3 M. H. Chiu, S. F. Wang and R. S. Chang: Appl. Opt. 43 (2004) 5438.

3 P. S. Huang, S. Kiyono and O. Kamada: Appl. Opt. 31 (1992) 6047.

4 P. S. Huang and J. Ni: Appl. Opt. 35 (1996) 2239.

5 S. F. Wang, M. H. Chiu, L. H. Shyu and R. S. Chang: Sens. Trans. 92 (2008) 16.

6 S. F. Wang, M. H. Chiu, C. W. Lai and R. S. Chang: Appl. Opt. 45 (2006) 6702.

7 L. Perumal: Int. J. Eng. Tech. Innovation (IJETI) 1 (2011) 35.

8 S. F. Wang: Sensors (Basel) 9 (2009) 2498.

9 M. Born and E. Wolf: Principles of Optics, 7th ed. (Cambridge University Press, Cambridge, 1999) Chap. 1.

10 E. Kretshmann: Z. Phys. 241 (1971) 313. 